\title{
A CHARACTERIZATION OF MINIMAL SURFACES IN ISOTHERMIC REPRESENTATION
}

\author{
MAXWELL O. READE ${ }^{1}$
}

1. Introduction. The real functions

$$
x_{j} \equiv x_{j}(u, v) \equiv x_{j}(z), \quad j=1,2,3,
$$

defined and continuous in the unit disc $\mathscr{D}:|z|<1$ will be said to define a surface $S$. In this note we shall study the equation

$$
\sum_{j=1}^{3}\left[\iint_{D(z, r)}(\zeta-z) x_{j}(\zeta) d \xi d \eta\right]^{2}=0, \quad \zeta=\xi+i \eta,
$$

where $D(z, r)$ denotes the closed disc in $\mathscr{D}$ with center $z$ and radius $r$. The equation (2) may be considered to be a generalization of a familiar equation of Féderoff $\left[3\right.$, p. 512]. ${ }^{2}$

If the first partial derivatives of the functions (1) are continuous and satisfy

$$
E(u, v)=G(u, v), \quad F(u, v)=0
$$

in $\mathscr{D}$, where

$$
\begin{gathered}
E(u, v) \equiv \sum_{j=1}^{3}\left(\frac{\partial x_{i}}{\partial u}\right)^{2}, \quad F(u, v) \equiv \sum_{j=1}^{3}\left(\frac{\partial x_{j}}{\partial u} \frac{\partial x_{j}}{\partial v}\right), \\
G(u, v) \equiv \sum_{j=1}^{3}\left(\frac{\partial x_{j}}{\partial v}\right)^{2}
\end{gathered}
$$

are the coefficients of the first fundamental differential quadratic form of $S$, then the parameters $u, v$ are said to be isothermic parameters, and $S$ is said to be given in isothermic representation. If (3) holds, then the map of $\mathcal{D}$ on $S$ is conformal except where $E=G=0$. If $E G-F^{2} \neq 0$ in $\mathcal{D}$, then we say $S$ is regular. From (4) it follows that (3) may be written in the form

$$
\sum_{j=1}^{8}\left(\lambda x_{j}\right)^{2}=0,
$$

where $\lambda \equiv \partial / \partial u+i \partial / \partial v$ is a differential operator.

Presented to the Society, December 30,1948; received by the editors October 21, 1949 and, in revised form, December 19, 1949.

${ }^{1}$ The author gratefully acknowledges aid provided by ERI of the University of Michigan under contract N8-ONR-581, Project M786.

${ }^{2}$ Numbers in brackets refer to the bibliography at the end of the paper. 
If the functions (1) are harmonic and satisfy (5) in $\mathcal{D}$, then they have been called a triple of conjugate harmonic functions [1]. In terms of these triples, a familiar theorem of Weierstrass may be stated as follows.

THEOREM 1. A necessary and sufficient condition that the functions (1), defined in $\mathcal{D}$, be the coordinate functions of a minimal surface in isothermic representation is that they constitute a triple of conjugate harmonic functions.

2. Principal results. We shall make use of the following result.

LEMMA. If the functions (1) are not identically constant in $\mathcal{D}$, if they have continuous partial derivatives of the third order in $\mathcal{D}$, and if they are the coordinate functions of a regular surface $S$, then a necessary and sufficient condition that they map $\mathcal{D}$ isothermically on a surface that lies on a sphere $S$ of finite non-null radius is that

$$
\sum_{j=1}^{3}\left[\iint_{D(z, r)}(\zeta-z) x_{j}(\zeta) d \xi d \eta\right]^{2}=o\left(r^{6}\right)
$$

hold at each point $z$ in $\mathscr{D}$, and that

$$
\sum_{j=1}^{3}\left[\iint_{D\left(z_{1}, r\right)}\left(\zeta-z_{1}\right) x_{j}(\zeta) d \xi d \eta\right]^{2} \neq o\left(r^{8}\right)
$$

hold at some point $z_{1}$ of $\mathcal{D}$.

Necessity. If the functions (1) map $D$ isothermically on a spherical surface $S$, then it follows from the formulas of Gauss [5, p. 359] that the functions (1) have continuous partial derivatives of all orders in $\mathscr{D}$. Hence, if we use Taylor expansions, we find

$$
\begin{aligned}
\sum_{j=1}^{3}\left[\iint_{D(z, r)}(\zeta\right. & \left.-z) x_{j}(\zeta) d \xi d \eta\right]^{2} \\
= & \frac{\pi^{2} r^{4}}{16} \sum_{j=1}^{3}\left(\lambda x_{j}\right)^{2}+\frac{\pi^{2} r^{6}}{96} \sum_{j=1}^{3} \lambda x_{j} \cdot \Delta \lambda x_{j} \\
& +\frac{\pi^{2} r^{8}}{9216} \sum_{j=1}^{3}\left(3 \lambda x_{j} \cdot \Delta^{2} \lambda x_{j}+4\left(\Delta \lambda x_{j}\right)^{2}\right)+o\left(r^{8}\right)
\end{aligned}
$$

where $\Delta \equiv \partial^{2} / \partial u^{2}+\partial^{2} / \partial v^{2}$ is a differential operator. Moreover it is also known that for this representation of $S$,

$$
\sum_{j=1}^{3} \lambda x_{j} \cdot \Delta \lambda x_{j}=0
$$


holds throughout $\mathcal{D}[5$, p. 361]. From (5), (8), and (9) it follows that (6) holds throughout $D$.

To show that there exist points at which (7) holds, we shall use a counterpositive proof. Suppose that

$$
\sum_{j=0}^{3}\left[\iint_{D(z, r)}(\zeta-z) x_{j}(\zeta) d \xi d \eta\right]^{2}=o\left(r^{8}\right)
$$

holds at each point of $\mathscr{D}$. Then it follows from (8) that

$$
\sum_{j=1}^{3}\left(3 \lambda x_{j} \cdot \Delta^{2} \lambda x_{j}+4\left(\Delta \lambda x_{j}\right)^{2}\right)=0
$$

holds throughout $\mathcal{D}$. But for $S$ it is known $[5$, p. 359] that

$$
\sum_{j=1}^{3} \lambda x_{j} \Delta^{2} \lambda x_{j}=-4 \alpha^{2} E \lambda^{2} E, \quad \sum_{j=1}^{3}\left(\Delta \lambda x_{j}\right)^{2}=4 \alpha^{2}(\lambda E)^{2}, \quad \alpha \neq 0,
$$

where $\alpha$ is a real constant. From (11) and (12) we obtain

$$
3 E \lambda^{2} E-4(\lambda E)^{2}=0 .
$$

Now we want to show the existence of an open subset $\mathscr{D}^{*}$ of $\mathscr{D}$ where $\lambda E \neq 0$ and $E \neq 0$. First, if $E$ were identically constant in $D$, then $S$ would be a plane surface, contrary to hypothesis. Hence there is an open subset $\mathscr{D}_{1}$ or $\mathscr{D}$ where $\lambda E \neq 0$. Second, if $E=0$ throughout $\mathcal{D}_{1}$, then $\lambda E=0$ there. Hence there is an open subset of $\mathscr{D}_{1}$ where $\lambda E \neq 0$ and $E \neq 0$, and this subset is denoted by $\mathcal{D}^{*}$.

For $z$ in $D^{*},(13)$ yields $3 \lambda \log (\lambda E)=4 \lambda(\log E)$, and hence

$$
\lambda E=E^{4 / 3} \Phi(z)
$$

where

$$
\lambda \Phi(z)=0 .
$$

From the imaginary part of (13) we obtain

$$
3 \frac{E_{u v}}{E_{u}} d v=4 \frac{E_{v}}{E} d v, \quad 3 \frac{E_{u v}}{E_{v}}=4 \frac{E_{u}}{E} d u,
$$

and hence

$$
E_{u}=E^{4 / 3} \Phi_{1}(u), \quad E_{v}=E^{4 / 3} \Phi_{2}(v),
$$

where $\Phi_{1}(u)$ and $\Phi_{2}(v)$ are real functions of $u$ and $v$, respectively. From (14), (15), and (16) we obtain

$$
\Phi(z) \equiv \Phi_{1}(u)+i \Phi_{2}(v),
$$


which, by (15), is an analytic function for $z$ in $\mathscr{D}^{*}$. If we use the Cauchy-Riemann equations for (17), then we find

$$
\Phi_{1}(u) \equiv 2 a_{0} u+a_{1}, \quad \Phi_{2}(v) \equiv 2 a_{0} v+a_{2},
$$

where $a_{0}, a_{1}, a_{2}$ are real constants. Therefore (14) yields

$$
\frac{E_{u}}{E^{4 / 3}} d u=\left(2 a_{0} u+a_{1}\right) d u, \quad \frac{E_{v}}{E^{4 / 3}} d v=\left(2 a_{0} v+a_{2}\right) d v,
$$

from which we obtain

$$
E=-\frac{27}{\left[a_{0}\left(u^{2}+v^{2}\right)+a_{1} u+a_{2} v+a_{3}\right]^{3}},
$$

where $a_{3}$ is another real constant. But it is well known that the Gaussian curvature of $S$ is given by [2]

$$
K=-\frac{1}{2 E} \Delta(\log E) \text {. }
$$

From (18) and (19), we find

$$
K=\frac{a_{1}^{2}+a_{2}^{2}-4 a_{0} a_{3}}{18}\left[a_{0}\left(u^{2}+v^{2}\right)+a_{1} u+a_{2} v+a_{3}\right],
$$

which holds throughout $D^{*}$. But $S$ is on the sphere $S$, so that (20) must be identically constant in $\mathscr{D}^{*}$. Hence $a_{0}=a_{1}=a_{2}=0$ and therefore $K=0$; therefore $D^{*}$ is mapped on a spherical surface with vanishing curvature. But $S$ has finite radius, so that we have been led to a contradiction by assuming (10) to hold throughout $\mathcal{D}$. Hence (7) holds for at least one point of, and hence in an open subset of, $\mathscr{D}$.

Sufficiency. By the use of finite Taylor expansions we obtain

$$
\begin{aligned}
\sum_{j=1}^{3}\left[\iint_{D(z, r)}(\zeta\right. & \left.-z) x_{j}(\zeta) d \xi d \eta\right]^{2} \\
= & \frac{\pi^{2} r^{4}}{16} \sum_{j=1}^{3}\left(\lambda x_{j}\right)^{2}+\frac{\pi^{2} r^{6}}{96} \sum_{j=1}^{3} \lambda x_{j} \Delta \lambda x_{j}+o\left(r^{6}\right) .
\end{aligned}
$$

From (6) and (21) we obtain (5) and (9). If we operate on (5) with the operator $\bar{\lambda}$, we obtain

$$
\sum_{j=1}^{3} \lambda x_{j} \Delta x_{j}=0
$$

Operating on (22) with $\lambda$, and applying (9) to the result, we obtain 


$$
\sum_{j=1}^{3} \lambda^{2} x_{j} \Delta x_{j}=0 .
$$

There are four real, linear, homogeneous equations in $\Delta x_{j}, j=1,2,3$, implied by (22) and (23):

$$
\begin{array}{ll}
\sum_{j=1}^{3} x_{j, u} \Delta x_{j}=0, & \sum_{j=1}^{3} x_{j, v} \Delta x_{j}=0, \\
\sum_{j=1}^{3}\left(x_{j, u u}-x_{j, v v}\right) \Delta x_{j}=0, & \sum_{j=1}^{3} x_{j, u v} \Delta x_{j}=0 .
\end{array}
$$

One solution to the system (24) is

$$
\Delta x_{j} \equiv 0, \quad j=1,2,3 .
$$

In this case the functions (1) are harmonic in $\mathcal{D}$, and hence (8) yields (10) for each $D(z, r)$ in $\mathscr{D}$. This contradicts (7). Hence the set $\mathcal{D}^{* *}$,

$$
\mathcal{D}^{* *} \equiv\left[z \mid z \in \mathcal{D} ; \sum_{j=1}^{3}\left(\Delta x_{j}\right)^{2} \neq 0 ;(24) \text { holds }\right],
$$

is a non-null open subset of $D$.

For $z$ in $\mathscr{D}^{* *}$, the system (24) has a nontrivial solution. Therefore the rank of the matrix

$$
\left\|\begin{array}{ccc}
x_{1, u} & x_{2, u} & x_{3, u} \\
x_{1, v} & x_{2, v} & x_{3, v} \\
x_{1, u u}-x_{1, v v} & x_{2, u u}-x_{2, v v} & x_{3, u u}-x_{8, v v} \\
x_{1, u v} & x_{2, u v} & x_{3, u v}
\end{array}\right\|
$$

is less than three. Hence it follows from the definitions of the coefficients $e, f, g$, of the second fundamental differential quadratic form of $S$, that

$$
e=g, \quad f=0,
$$

holds in $D^{* *}$. Hence it follows from (5) and (26) that the functions (1) map $\mathscr{D}^{* *}$ isothermically on a spherical surface $S^{* *}$. If $S^{* *}$ were either a plane or a point, then (25) would hold in $\mathcal{D}^{* *}$; hence $S^{* *}$ lies on a sphere of finite non-null radius.

Now consider the subset $\mathcal{D}-\mathscr{D}^{* *}$ of $\mathcal{D}$, and let $z_{0}=u_{0}+i v_{0}$ be a point of $\mathscr{D}-\mathscr{D}^{* *}$. If $z_{0}$ is a frontier point of $\mathscr{D}-\mathscr{D}^{* *}$, then a simple continuity argument shows that the functions (1) map $z_{0}$ on the boundary of $S^{* *}$, lying on the same sphere containing $S^{* *}$; moreover, since $S$ is regular, we know that $E\left(u_{0}, v_{0}\right) \neq 0$, so that the con- 
tinuity of the Gaussian curvature shows that $K\left(u_{0}, v_{0}\right)>0$. Now if $\mathcal{D}-\mathcal{D}^{* *}$ has a component $\mathcal{C}$ with non-null interior, then the functions (1) map this interior on a minimal surface $\mathcal{H}$, such that the frontier of $\mathcal{C}$ is mapped on the boundary of $\mathcal{X}$. Since a minimal surface is a surface of nonpositive Gaussian curvature, it follows by a continuity argument again that the Gaussian curvature at points of the boundary of $\mathcal{H}$ satisfy $K\left(u_{0}, v_{0}\right) \leqq 0$. But at the beginning of this paragraph it was pointed out that $K\left(u_{0}, v_{0}\right)>0$ at the points corrresponding to frontier points of $\mathscr{D}-\mathcal{D}^{* *}$. Since $S^{* *}$ and $\mathcal{X}$ are merely parts of the regular surface $S$, it follows from this contradiction that the set $\mathcal{D}-\mathscr{D}^{* *}$ has no component with a non-null interior, and hence all points $\mathcal{D}-D^{* *}$ are mapped on the boundary of $S^{* *}$. Therefore the functions (1) map all of $\mathcal{D}$ isothermically on a spherical surface, lying on a sphere with finite non-null radius.

The following result does not appear to demand the regularity of the surfaces involved.

THEOREM 2. If the functions(1) have continuous partial derivatives of the third order in $D$, then a necessary and sufficient condition that they be the coordinate functions of a minimal surface in isothermic representation is that

$$
\sum_{j=1}^{3}\left[\iint_{D(z, r)}(\zeta-z) x_{j}(\zeta) d \xi d \eta\right]^{2}=0
$$

hold for each $D(z, r)$ in $\mathcal{D}$.

Necessity. If the functions (1) map $\mathcal{D}$ isothermically on a minimal surface, then it follows from Theorem 1 that (5) and (25) hold, and hence, by the use of Fourier expansions, it follows that (27) holds for each $D(z, r)$ in $D$.

Sufficiency. If (27) holds, then (5), (22), and (23) follow from (21). Hence we obtain the system (24). Now consider the set $D^{* *}$ defined in the proof of the lemma. As in the proof, the functions (1) map $\mathscr{D}^{* *}$ isothermically on a surface $S^{* *}$ that lies on a sphere with finite nonnull radius. Hence the functions (1) are analytic in the real variables $u, v[5$, p. 358]. Therefore we may use (8) to obtain (11). Hence we find that (18) holds in $\mathscr{D}^{* *}$, and consequently, as in the proof of the necessity part of the lemma, the Gaussian curvature of $S^{* *}$ is identically zero. Therefore the spherical surface $S^{* *}$ is a plane surface, so that (25) holds in $\mathscr{D}^{* *}$. From this contradiction we conclude that the open set $\mathscr{D}^{* *}$ contains no interior points, hence is a null set. Therefore (5) and (25) hold throughout $\mathcal{D}$, and hence $S$ is a minimal surface given in isothermic representation by the functions (1). 
CoROLlaRY. If the functions (1) have continuous partial derivatives of the third order in $\mathcal{D}$, then a necessary and sufficient condition that they be the coordinate functions of a minimal surface in isothermic representation is that (10) hold at each point of $\mathcal{D}$.

3. Characterization of plane isothermic maps. We take this occasion to offer a simplification of a proof of a characterization of plane isothermic maps, a result which may be considered to be a generalization to space of the Cauchy and Morera theorems. The simplification consists in deriving some fundamental identities without the use of conformal mapping and schlicht functions [5, pp. 368-370].

THEOREM 3. If the functions (1) have continuous partial derivatives of the third order in $\mathcal{D}$, then a necessary and sufficient condition that they be the coordinate functions of a plane surface in isothermic representation is that

$$
\sum_{j=1}^{3}\left[\int_{\gamma} x_{j}(\zeta) d \zeta\right]^{2}=0
$$

hold for each closed rectifiable Jordan curve $\gamma$ in $\mathscr{D}$.

Proof. In the earlier proof it was shown that (28) is a necessary condition that the functions (1) map $\mathcal{D}$ isothermically on a plane surface. It was also shown that if (28) holds for all closed rectifiable Jordan curves $\gamma$ in $\mathcal{D}$, then the functions (1) constitute a triple of conjugate harmonic functions in $\mathcal{D}$ and hence may be written in the form

$$
x_{j}(z) \equiv F_{j}(z)+\overline{F_{j}(z),} \quad j=1,2,3,
$$

where $F_{j}(z)$ is analytic in $\mathscr{D}$ and where $\overline{F_{j}(z)}$ is its complex conjugate.

Now let $z=u+i v$ be an arbitrary point in $\mathcal{D}$, and write

$$
F_{j}(\zeta)=\sum_{n=0}^{\infty} a_{j, n}(\zeta-z)^{n}, \quad \overline{F_{j}(z)}=\sum_{n=0}^{\infty} \bar{a}_{j, n}\left(\overline{\zeta-z)^{n}} .\right.
$$

Now consider the family of triangles $\gamma(r, \alpha)$, with vertices $z, z+r e^{i \alpha}$, $z+r e^{-i \alpha}$. Then a direct computation, using (28) and (29), yields

$$
\begin{aligned}
& \sum_{j=1}^{3}\left[\int_{\gamma(r, \alpha)} x_{j}(\zeta) d \zeta\right]^{2} \\
& \quad=-4 \cos ^{2} \alpha \sum_{n=0}^{\infty} r^{n+1} \sum_{k=0}^{n} \sin k \alpha \sin (n-k) \alpha\left[\sum_{j=1}^{3} \bar{a}_{j, k} \bar{a}_{j, n-k}\right]=0,
\end{aligned}
$$

which must hold for $\gamma(r, \alpha)$ in $\mathscr{D}$. Since (30) holds for all sufficiently 
small $r$, it follows that

$$
\sum_{k=0}^{n} \sin k \alpha \sin (n-k) \alpha\left[\sum_{j=1}^{3} \bar{a}_{j, k} \bar{a}_{j, n-k}\right]=0, \quad n \geqq 0,
$$

holds for all $\alpha$. From (31) we obtain

$$
\sum_{k=1}^{n-1}[\cos (n-2 k) \alpha-\cos n \alpha]\left[\sum_{j=1}^{3} \bar{a}_{j, k} \bar{a}_{j, n-k}\right]=0, \quad n \geqq 2,
$$

which must hold for all $\alpha$. Now let $p$ be a fixed integer, $1 \leqq p \leqq n-1$. If we multiply both members of (32) by $\cos (n-2 p) \alpha$ and then integrate the result over the interval $(0,2 \pi)$, we obtain

$$
\bar{a}_{n-p}, \bar{a}_{n+p}=0, \quad 1 \leqq p \leqq n-1, n \geqq 2 .
$$

Now (33) are the identities that had to be proved in order to prove that the functions (1) map $\mathcal{D}$ isothermically on a plane surface.

4. Conclusion. The results contained in this note are similar to those obtained before [5]: The one outstanding point of difference is the lack of a characterization of isothermic plane maps by means of an equation similar to (5). It would be interesting to obtain such a characterization.

\section{BIBLIOGRAPHY}

1. E. F. Beckenbach and T. Rad6, Subharmonic functions and minimal surfaces, Trans. Amer. Math. Soc. vol. 35 (1933) pp. 648-661.

2. - Subharmonic functions and surfaces and surfaces of negative curvature, Trans. Amer. Math. Soc. vol. 35 (1933) pp. 662-674.

3. V. Fédoroff, Sur une proprieté caracteristique des fonctions monogènes, C. R. Acad. Sci. Paris vol. 193 (1931) pp. 512-513.

4. W. C. Graustein, Differential geometry, New York, 1935.

5. Maxwell Reade and E. F. Beckenbach, Generalizations to space of the Cauchy and Morera theorems, Trans. Amer. Math. Soc. vol. 49 (1941) pp. 354-377.

UNIVERSITY OF MichigAN 